\title{
Analytical Design of 2-D Narrow Bandstop FIR Filters
}

\author{
Pavel Zahradnik ${ }^{1}$ and Miroslav Vlček ${ }^{2}$ \\ 1 Department of Telecommunications Engineering \\ Czech Technical University Prague \\ Technická 2, CZ-166 27 Praha, Czech Republic \\ Phone: +420-2-24352089, Fax: +420-2-33339810 \\ zahradni@fel.cvut.cz \\ 2 Department of Applied Mathematics \\ Czech Technical University Prague \\ Konviktská 20, CZ-110 00 Praha, Czech Republic \\ Phone: +420-2-24890720, Fax:+420-2-24890702 \\ vlcek@fel.cvut.cz
}

\begin{abstract}
Novel approach in the design of 2-D extremely narrow bandstop FIR filters is presented. The completely analytical design method is based on the 1-D optimal bandstop FIR filters. The 1-D FIR optimal bandstop filters are based on Zolotarev polynomials. Closed form formulas for the design of the filters are presented. One example demonstrates the design procedure. One application of the 2-D FIR filter with extremely narrow stop bands is presented.
\end{abstract}

\section{Introduction}

Two-dimensional narrow bandstop FIR filters play important role in the image and video enhancement/restoration tasks. They are frequently used in order to remove a single frequency component from the spectrum of the signal. The image filtering can be accomplished by both the nonlinear [1], 4], 7], [8], [9], 10] and linear [5, 12, 13], 14, 15] filters. In our paper we are concerned with completely analytical design of 2-D bandstop FIR filters with extremely narrow circularly symmetrical stop bands. The design of the 2-D narrow bandstop FIR filters is based on the 1-D optimal narrow bandstop FIR filters 14. We introduce the degree formula which relates the degree of the generating polynomial, the length of the filter, the notch frequency, the width of the stopbands and the attenuation in the passbands. Based on the expansion of the generating polynomials into the Chebyshev polynomials, the recursive formula for the direct computation of the impulse response coefficients is presented. The design procedure is recursive one and it does not require any FFT algorithm or any iterative technique. 


\section{Polynomial Equiripple Approximation}

Let us denote $H(z)$ the transfer function of a 1-D FIR filter with the impulse response $h(m)$ of the length $N$ as

$$
H(z)=\sum_{m=0}^{N-1} h(m) z^{-m} .
$$

Assuming an odd length $N=2 n+1$ and even symmetry of the impulse response $h(m)$ we can write the transfer function of the bandstop FIR filter

$$
H(z)=z^{-n}\left[h(0)+\sum_{m=1}^{n} 2 h(m) T_{m}(w)\right]=z^{-n}\left[h(0)+\sum_{m=1}^{n} 2 h(m) T_{m}(\cos \omega T)\right]
$$

where $T_{m}(w)$ is Chebyshev polynomial of the first kind and $w=\left(z+z^{-1}\right) / 2$. The
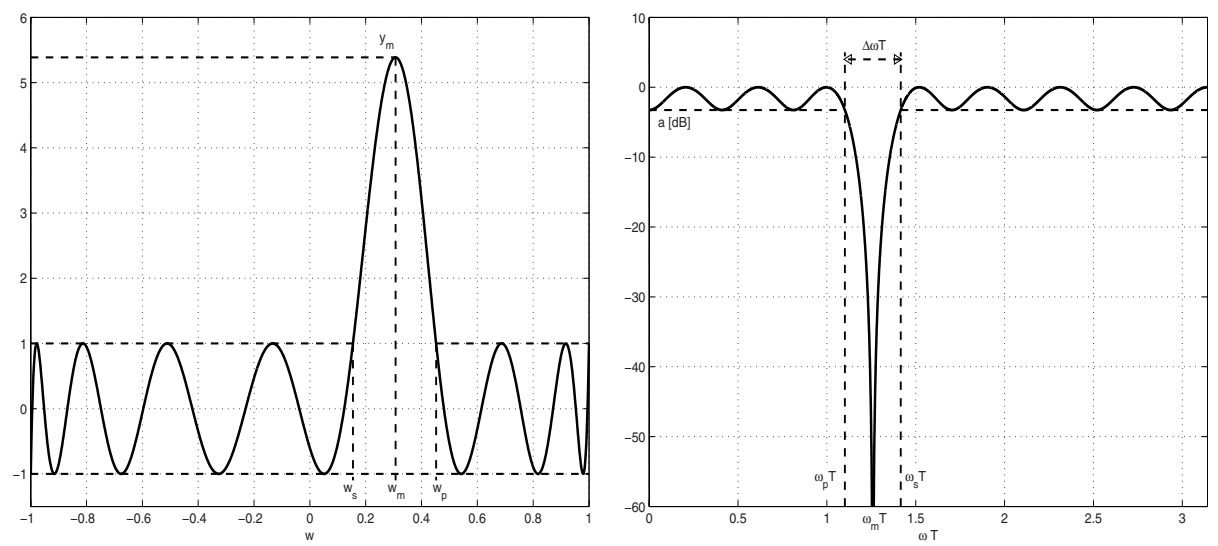

Fig. 1. Zolotarev polynomial $Z_{6,9}(w)$ with $\kappa=0.6966, w_{s}=0.1543, w_{m}=$ $0.3071, w_{p}=0.4523, y_{m}=5.3864$ and corresponding amplitude frequency response $20 \log \left|H\left(e^{j \omega T}\right)\right|[\mathrm{dB}]$ with parameters $\omega_{p} T=0.3506 \pi, \omega_{m} T=0.4006 \pi$, $\omega_{s} T=0.4507 \pi, \Delta \omega T=0.1001 \pi$ and $a=-3.2634 \mathrm{~dB}$

1-D equiripple narrow bandstop FIR filter is based on the Zolotarev polynomial $Z_{p, q}(w)$ which approximates constant value in equiripple Chebyshev sense in the two disjoint intervals as shown in Fig. 11. The notation $Z_{p, q}(w)$ emphasizes that $p$ counts the number of zeros right from the maximum $w_{m}$ and $q$ corresponds to the number of zeros left from the maximum $w_{m}$. Zolotarev derived the general solution of this approximation problem in terms of Jacobi's elliptic functions

$$
Z_{p, q}(w)=\frac{(-1)^{p}}{2}\left[\left(\frac{H\left(u-\frac{p}{n} \mathbf{K}(\kappa)\right)}{H\left(u+\frac{p}{n} \mathbf{K}(\kappa)\right)}\right)^{n}+\left(\frac{H\left(u+\frac{p}{n} \mathbf{K}(\kappa)\right)}{H\left(u-\frac{p}{n} \mathbf{K}(\kappa)\right)}\right)^{n}\right]
$$

where $u$ is expressed by the incomplete elliptical integral of the first kind 


$$
u=F\left(\operatorname{sn}\left(\frac{p}{n} \mathbf{K}(\kappa) \mid \kappa\right) \sqrt{\frac{1+w}{w+2 \operatorname{sn}^{2}\left(\frac{p}{n} \mathbf{K}(\kappa) \mid \kappa\right)-1}} \mid \kappa\right) .
$$

The function $H\left(u \pm \frac{p}{n} \mathbf{K}(\kappa)\right)$ is the Jacobi's Eta function, $\operatorname{sn}(u \mid \kappa), \operatorname{cn}(u \mid \kappa)$, $\operatorname{dn}(u \mid \kappa)$ are Jacobi's elliptic functions, $\mathbf{K}(\kappa)$ is the quarter-period given by the complete elliptic integral of the first kind, $F(\phi \mid \kappa)$ is the incomplete elliptic integral of the first kind and $\kappa$ is the Jacobi's elliptic modulus. The degree of the Zolotarev polynomial is $n=p+q$. A comprehensive treatise of the Zolotarev polynomials was published in [14]. It includes the analytical solution of the coefficients of Zolotarev polynomials, the algebraic evaluation of the Jacobi's Zeta function $\mathrm{Z}\left(\frac{p}{n} \mathbf{K}(\kappa) \mid \kappa\right)$ and of the elliptic integral of the third kind $\Pi\left(\sigma_{m}, \frac{p}{n} \mathbf{K}(\kappa) \mid \kappa\right)$ of the discrete argument. The position of the maximum value $y_{m}=Z_{p, q}\left(w_{m}\right)$ is

$$
w_{m}=1-2 \operatorname{sn}^{2}\left(\frac{p}{n} \mathbf{K}(\kappa) \mid \kappa\right)+2 \frac{\operatorname{sn}\left(\frac{p}{n} \mathbf{K}(\kappa) \mid \kappa\right) \operatorname{cn}\left(\frac{p}{n} \mathbf{K}(\kappa) \mid \kappa\right)}{\operatorname{dn}\left(\frac{p}{n} \mathbf{K}(\kappa) \mid \kappa\right)} \mathrm{Z}\left(\frac{p}{n} \mathbf{K}(\kappa) \mid \kappa\right) .
$$

The maximum value $y_{m}$ useful for the normalization of the Zolotarev polynomial is given as

$$
y_{m}=\cosh 2 n\left(\sigma_{m} \mathrm{Z}\left(\frac{p}{n} \mathbf{K}(\kappa) \mid \kappa\right)-\Pi\left(\sigma_{m}, \frac{p}{n} \mathbf{K}(\kappa) \mid \kappa\right)\right) .
$$

The degree of the Zolotarev polynomial $Z_{p, q}(w)$ expresses the degree equation

$$
n \geq \frac{\ln \left(y_{m}+\sqrt{y_{m}^{2}-1}\right)}{2 \sigma_{m} \mathrm{Z}\left(\frac{p}{n} \mathbf{K}(\kappa) \mid \kappa\right)-2 \Pi\left(\sigma_{m}, \frac{p}{n} \mathbf{K}(\kappa) \mid \kappa\right)} .
$$

The auxiliary parameter $\sigma_{m}$ is given by the formula

$$
\sigma_{m}=F\left(\arcsin \left(\frac{1}{\kappa \operatorname{sn}\left(\frac{p}{n} \mathbf{K}(\kappa) \mid \kappa\right)} \sqrt{\frac{w_{m}-w_{s}}{w_{m}+1}}\right) \mid \kappa\right)
$$

where $F(\Phi \mid \kappa)$ is the incomplete elliptical integral of the first kind. The recursive algorithm for the evaluation of the coefficients $a(m)$ of the Zolotarev polynomial based on the expansion into Chebyshev polynomials of the first kind

$$
Z_{p, q}(w)=\sum_{m=0}^{n} a(m) T_{m}(w)
$$

was derived and presented in [14. It is summarized in Table 1]. The impulse response coefficients $h(m)$ of the 1-D equiripple bandstop FIR filter are obtained by the normalization of the coefficients $a(m)$ as follows

$$
h(n)=\frac{y_{m}-a(0)}{y_{m}+1}, h(n \pm m)=-\frac{a(m)}{2\left(y_{m}+1\right)}, m=1 \ldots n .
$$




\section{Analytical Design of 2-D Narrow Bandstop FIR Filter}

The goal of the design of the 2-D narrow bandstop FIR filter is to obtain the 2$\mathrm{D}$ impulse response $h(m, n)$ of the filter satisfying the specified notch frequency $\omega_{m 1} T$, width of the bandstop $\Delta \omega_{1} T$, the attenuation in the passbands $a_{1}[\mathrm{~dB}]$ in the direction $\omega_{1}$ and the specified values $\omega_{m 2} T, \Delta \omega_{2} T, a_{2}[\mathrm{~dB}]$ in the direction $\omega_{2}$. The design procedure is as follows :

1. For the specified values $\omega_{m 1} T, \Delta \omega_{1} T$ and $a_{1}$ [dB] (Fig. 1) in the direction $\omega_{1}$ design the 1-D FIR narrow bandpass filter. The design procedure consists of the following steps :

a) Evaluate the Jacobi's elliptic modulus $\kappa$

$$
\kappa=\sqrt{1-\frac{1}{\tan ^{2}\left(\varphi_{s}\right) \tan ^{2}\left(\varphi_{p}\right)}}
$$

for the auxiliary parameters $\varphi_{s}$ and $\varphi_{p}$

$$
\varphi_{s}=\frac{\omega_{m 1}+\Delta \omega_{1} / 2}{2} T, \varphi_{p}=\frac{\pi-\left(\omega_{m 1}-\Delta \omega_{1} / 2\right)}{2} T .
$$

b) Calculate the rational values $\frac{p}{n}=\frac{F\left(\varphi_{s} \mid \kappa\right)}{\mathbf{K}(\kappa)}$ and $\frac{q}{n}=\frac{F\left(\varphi_{p} \mid \kappa\right)}{\mathbf{K}(\kappa)}$.

c) Determine the required maximum value $y_{m}$

$$
y_{m}=\frac{2}{1-10^{0.05 a_{1}[d B]}}-1 .
$$

d) Using the degree equation (7) calculate and round up the minimum degree $n$ required to satisfy the filter specification. For the algebraic evaluation of the Jacobi's Zeta function $\mathrm{Z}\left(\frac{p}{n} \mathbf{K}(\kappa) \mid \kappa\right)$ and the elliptic integral of the third kind $\Pi\left(\sigma_{m}, \frac{p}{n} \mathbf{K}(\kappa) \mid \kappa\right)$ in the degree equation (7) use the algebraical procedure [14].

e) Calculate the integer values $p=\left[n \frac{F\left(\varphi_{s} \mid \kappa\right)}{\mathbf{K}(\kappa)}\right]$ and $q=\left[n \frac{F\left(\varphi_{p} \mid \kappa\right)}{\mathbf{K}(\kappa)}\right]$. The brackets [ ] stand for the rounding operation.

f) For the integer values $p, q$ and the elliptic modulus $\kappa$ evaluate the coefficients $a(m)$ (9) of the Zolotarev polynomial $Z_{p, q}(w)$ using recursive algorithm summarized in Tab. 1

g) From the coefficients $a(m)$ calculate the $M$ coefficients of the impulse response $h_{1}(m)$ of the 1-D equiripple bandpass FIR filter using (10).

2. Repeat the first step for the design of the 1-D FIR equiripple narrow bandpass filter in the direction $\omega_{2}$ specified by $\omega_{m 2} T, \Delta \omega_{2} T$ and $a_{2}$ [dB] resulting in the impulse response $h_{2}(n)$ of the length $N$ coefficients.

3. From the 1-D impulse responses

$$
h_{1}(m), \quad m=0, \ldots, M-1 \quad, \quad h_{2}(n), \quad n=0, \ldots, N-1
$$


compose the 2-D impulse responses $h_{1}(m, n)$ and $h_{2}(m, n)$ by the zero padding. The non-zero coefficients are

$$
\begin{aligned}
& h_{1}\left(\frac{M-1}{2}, n\right)=h_{1}(m), \quad m=0, \ldots, M-1 \\
& h_{2}\left(m, \frac{N-1}{2}\right)=h_{2}(n), \quad n=0, \ldots, N-1 .
\end{aligned}
$$

4. The 2-D impulse response $h_{B P}(m, n)$ of the dimension $M \times N$ of the narrow bandpass FIR filter is given by the 2-D linear discrete convolution

$$
h_{B P}(m, n)=h_{1}(m, n) * * h_{2}(m, n) .
$$

5. The impulse response $h(m, n)$ of the final 2-D bandstop FIR filter is

$$
\begin{array}{r}
h(m, n)=-h_{B P}(m, n) \text { for } m \neq \frac{M-1}{2} \\
n \neq \frac{N-1}{2} \\
h\left(\frac{M-1}{2}, \frac{N-1}{2}\right)=1-h_{B P}\left(\frac{M-1}{2}, \frac{N-1}{2}\right) .
\end{array}
$$

\section{Example}

Design the 2-D bandstop FIR filter specified in the direction $\omega_{1}$ by the notch frequency $\omega_{m 1} T=0.4 \pi$, width of the passbands $\Delta \omega_{1} T=0.1 \pi$ for the attenuation in the passbands $a_{1}=-1 \mathrm{~dB}$ and in the direction $\omega_{2}$ by the values $\omega_{m 2} T=0.6 \pi$, $\Delta \omega_{2} T=0.1 \pi$ for $a_{2}=-1 \mathrm{~dB}$.

Using our recursive design procedure we obtain the two 1-D equiripple narrow band FIR filters with the impulse responses $h_{1}(m), h_{2}(n)$ (step 1 and 2 in Sec. 3). The impulse responses $h_{1}(m), h_{2}(n)$ of the length $M=N=41$ coefficients are summarized in Table 2 Their amplitude frequency responses are shown in Fig. 2. The impulse responses $h_{1}(m), h_{2}(n)$ are used for the design of the 2-D bandstop FIR filter (step 3, 4 and 5 in Sec. [3). The impulse response $h(m, n)$ of the 2 -D narrow bandstop FIR filter consists of $41 \times 41$ coefficients. The amplitude frequency response $20 \log \left|H\left(e^{j \omega_{1}}, e^{j \omega_{2}}\right)\right|$ of the 2-D narrow bandstop FIR filter with its contours is shown in Fig. 3

\section{Application of the 2-D Narrow Bandstop FIR Filter}

The narrow 2-D bandstop FIR filters were successfully applied for the removal of the unwanted frequency components in the spectrum of the image. Here we present the enhancement of the rastered newspaper picture. The notch frequencies $\omega_{1}=0.32 \pi, \omega_{2}=0.42 \pi$ to be removed were obtained by the evaluation of the spectrum of the input image. The impulse response $h(m, n)$ of the applied filter exhibits $37 \times 37$ coefficients. The input and processed image are shown in Fig. 4. The attenuation of the disturbing raster is apparent. 

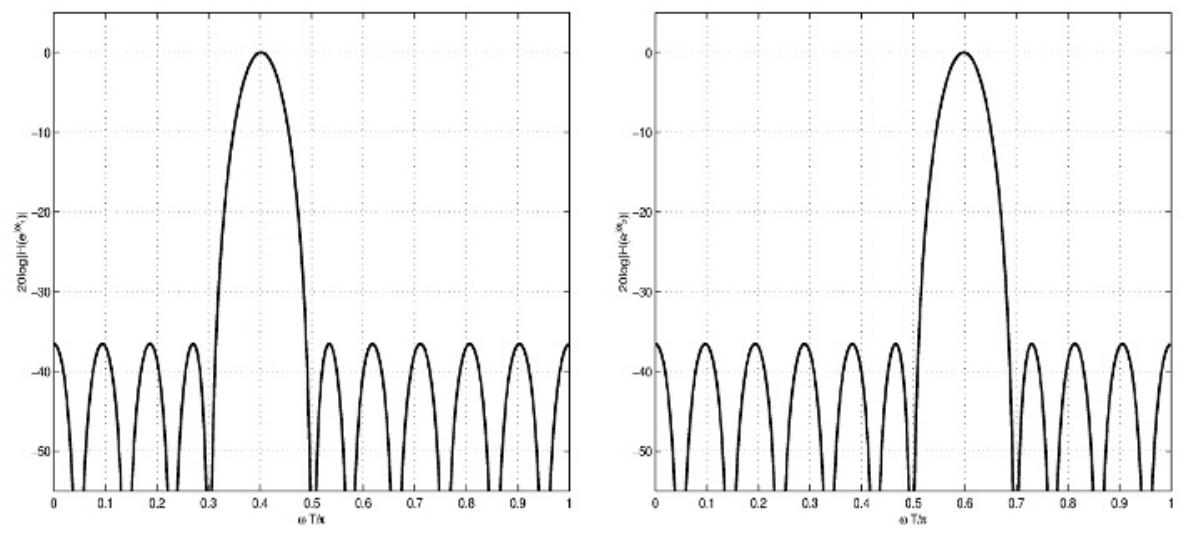

Fig. 2. Amplitude frequency responses $20 \log \left|H\left(e^{j \omega_{1}}\right)\right|$ and $20 \log \left|H\left(e^{j \omega_{2}}\right)\right|$
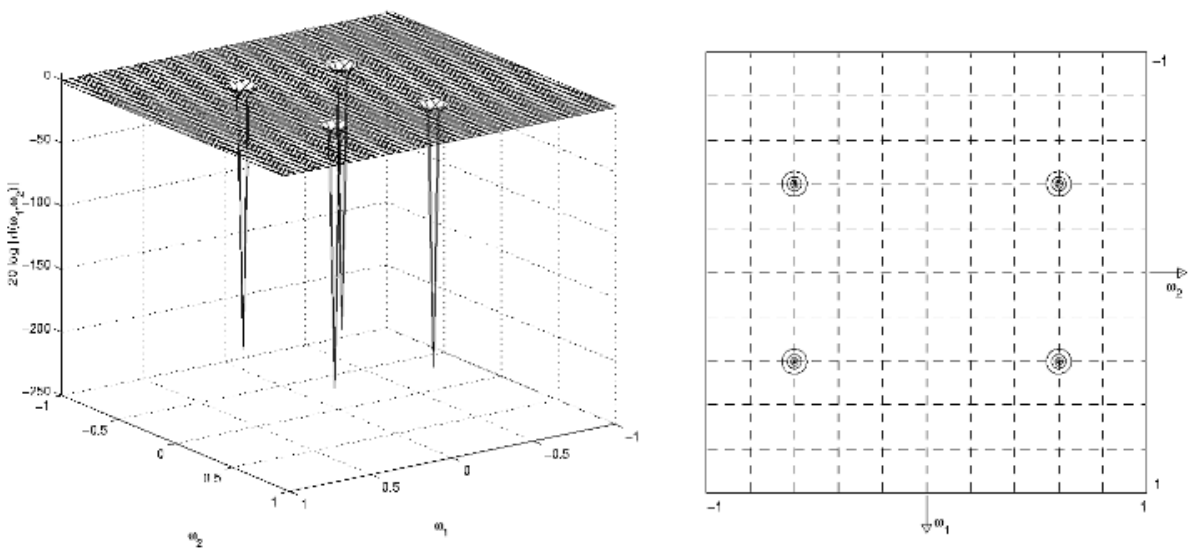

Fig. 3. Amplitude frequency response $\left|H\left(e^{j \omega_{1}}, e^{j \omega_{2}}\right)\right|$ with contours
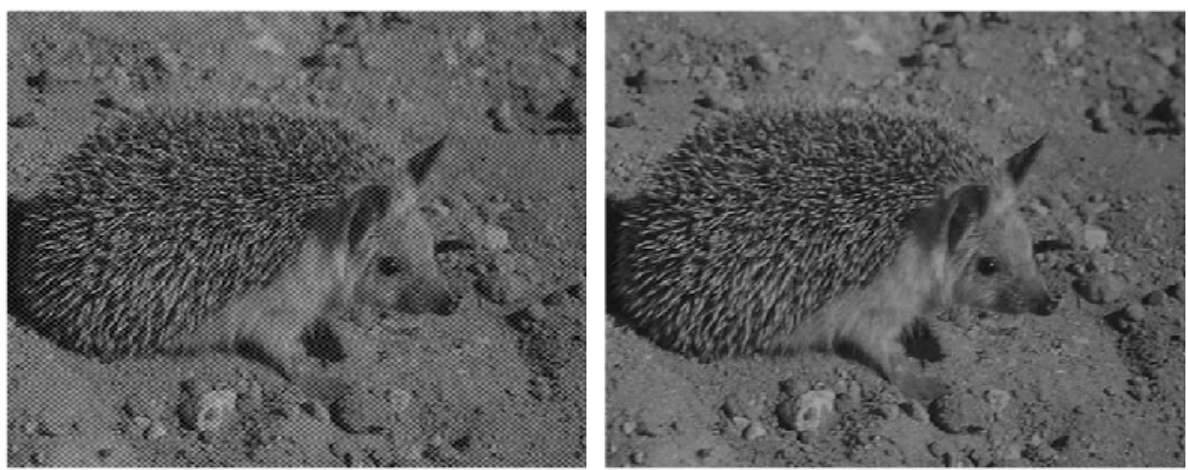

Fig. 4. Input and filtered image 
Table 1. Recursive algorithm for the evaluation of the coefficients $a(m)$ of the Zolotarev polynomials $Z_{p, q}(w)$

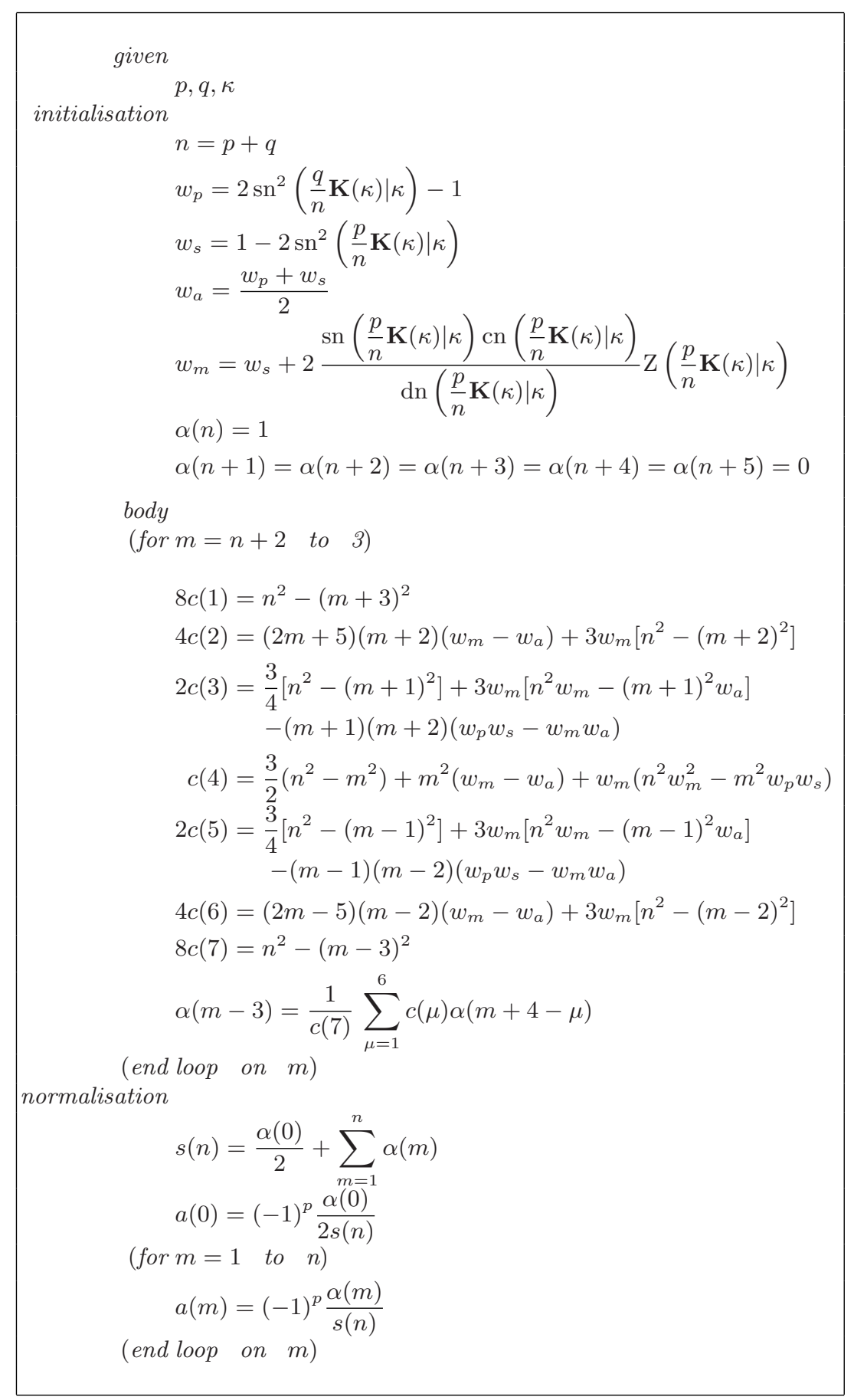


Table 2. Coefficients of the Impulse Responses

\begin{tabular}{|c|c|c|c|c|c|c|c|}
\hline \multicolumn{2}{|c|}{$m, n$} & \multirow{2}{*}{$\begin{array}{c}h_{1}(m) \\
0.008036\end{array}$} & \multirow{2}{*}{$\begin{array}{c}h_{2}(n) \\
0.008036\end{array}$} & \multicolumn{2}{|c|}{$m, n$} & \multirow{2}{*}{$\begin{array}{c}h_{1}(m) \\
0.020208\end{array}$} & \multirow{2}{*}{$\begin{array}{c}h_{2}(n) \\
-0.020208\end{array}$} \\
\hline 0 & 40 & & & 11 & 29 & & \\
\hline 1 & 39 & 0.003713 & -0.003713 & 12 & 28 & -0.047824 & -0.047824 \\
\hline 2 & 38 & -0.008856 & -0.008856 & 13 & 27 & -0.055411 & 0.055411 \\
\hline 3 & 37 & -0.013403 & 0.013403 & 14 & 26 & 0.019492 & 0.019492 \\
\hline 4 & 36 & 0.004187 & 0.004187 & 15 & 25 & 0.075345 & -0.075345 \\
\hline 5 & 35 & 0.023801 & -0.023801 & 16 & 24 & 0.026236 & 0.026236 \\
\hline 6 & 34 & 0.011174 & 0.011174 & 17 & 23 & -0.065318 & 0.065318 \\
\hline 7 & 33 & -0.025726 & 0.025726 & 18 & 22 & -0.068525 & -0.068525 \\
\hline 8 & 32 & -0.033363 & -0.033363 & 19 & 21 & 0.025845 & -0.025845 \\
\hline 9 & 31 & 0.010947 & -0.010947 & & & 0.093129 & 0.093129 \\
\hline 10 & 30 & 0.050326 & 0.050326 & & & & \\
\hline
\end{tabular}

\section{References}

1. Astola J., Kuosmanen P.: Fundamentals of Nonlinear Digital Filtering. CRC Press, 1997.

2. Abramowitz M., Stegun I.: Handbook of Mathematical Function. Dover Publication, New York Inc., 1972.

3. Achieser N. I.: Über einige Funktionen, die in gegebenen Intervallen am wenigsten von Null abweichen. Bull. de la Soc. Phys. Math. de Kazan, Vol. 3, pp. 1 - 69, 1928.

4. Fischer V., Drutarovsky M., Lukac R.: Implementation of 3-D Adaptive LUM Smoother in Reconfigurable Hardware. Springer Verlag LNCS 2438, pp.720-729.

5. Gonzales R.C., Woods R.E.: Digital Image processing. Wiley Interscience, 2001.

6. Lawden D. F.: Elliptic Functions and Applications. Springer-Verlag, New York Inc., 1989.

7. Lukac R.: Binary LUM Smoothing. IEEE Signal Processing Letters, Vol. 9, No. 12, December 2002, pp. 400-403.

8. Lukac R.: Adaptive Vector Median Filtering. Pattern Recognition Letters, Vol. 24, No. 12, August 2003, pp. 1889-1899.

9. Lukac R.: Simplified Boolean LUM Smoothers. Proceedings of the 4th EURASIPIEEE Region 8 International Symposium on Video/Image Processing and Multimedia Communications VIPromCom-2002, Zadar, Croatia, June 16-19, 2002, pp. 159-162.

10. Lukac R.: The Way How to Design and Implement an Adaptive Method Based on Center-Weighted Medians. Proceedings of the IEEE Scientific Workshop Signal Processing 2002, Poznan, Poland, October 11, 2002, pp.9-14.

11. Pitas I., Venetsanopoulos A.N.: Nonlinear Digital Filters : Priciples and Applications. Kluwer Academic Publishers, 1990.

12. Pratt W. K., Venetsanopoulos A.N.: Digital Image processing. Kluwer Academic Publishers, 1990.

13. Vlček M., Jireš L.: Fast Design Algorithms for FIR Notch Filters. Proc. of IEEE International Symposium on Circuits and Systems ISCAS'94, London, Vol. 2, pp. $297-300,1994$.

14. Vlček M., Unbehauen R.: Zolotarev Polynomials and Optimal FIR Filters. IEEE Transactions on Signal Processing, Vol. 47, No. 3, pp. 717-730, March 1999.

15. Vlček M., Zahradnik P., Unbehauen R.: Analytic Design of FIR Filters. IEEE Transactions on Signal Processing, Vol. 48, pp. 2705-2709, September 2000. 